\title{
Cardiopatías congénitas, tratamiento quirúrgico y sus complicaciones en población pediátrica del Hospital Vicente Corral Moscoso, 2017- Agosto 2019
}

\author{
Jennifer Pamela Picón Rodríguez ${ }^{1}$, Luis Enrique Marcano Sanz², Erika Aracely Pinos Vélez ${ }^{1}$,Susana \\ Janeth Peña Cordero ${ }^{3}$, Carlos Eduardo Arévalo Peláez $z^{3-4}$.
}

\begin{abstract}
1. Clínica Latinoamericana, Cuenca, Ecuador. 2. Servicio de Cardiología Pediátrica, Hospital Vicente Corral Moscoso. Cuenca, Ecuador.

3. Unidad Académica de Salud y Bienestar de la Universidad Católica de Cuenca, Ecuador.

4. Facultad de Ciencias Médicas, Universidad de

Cuenca. Ecuador.
\end{abstract}

\section{CORRESPONDENCIA:}

Jennifer Pamela Picón Rodríguez

jerreo electrónico:

jennifer2436

Francisco de Orellana 469 y Av. Don Bosco.

Código Postal: 010105

Teléfono: 0987169045 / 2887193.

Fecha de Recepción: 09-02-2021.

Fecha de Aceptación: 14-03-202

Fecha de Publicación: 30-03-2021.

\section{MEMBRETE BIBLIOGRÁFICO:}

Picón J, Marcano L, Pinos E, Peña S, Arévalo C. Cardiopatías congénitas, tratamiento quirúrgico y sus complicaciones en población pediátrica del Hospital Vicente Corral Moscoso, 2017- Agosto 2019. Rev Med HJCA 2021; 13(1): 21-26. DOI: http://dx.doi.org/10.14410/2021.13.1.ao.03

\section{ARTÍCULO ACCESO ABIERTO}

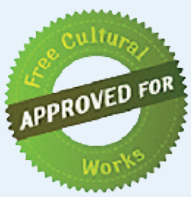

O2021 Picón et al. Licencia RevMed HJCA. Este es un artículo de acceso abierto distribuido bajo los términos de "Creative Commons Attribution-NonCommercial-ShareAlike 4.0 International Public License" (http://creativecommons.org/licenses/by-nc-sa/4.0/), la cual permite copiar y redistribuir el material en cualquier medio o formato; mezclar, transformar y crear a partir del material, dando el crédito adecuado al propietario del trabajo original y bajo la misma licencia del original.

El dominio público de transferencia de propiens.org/publicdomain/zero/1.0/) aplica a los datos recolectados y disponibles en este artículo, a no se que exista otra disposición del autor.

* Cada término de los Descriptores de Ciencias de la Salud (DeCS) reportados en este artículo ha sido verificado por el editor en la Biblioteca Virtual de Salud (BVS) de la edición actualizada a marzo de 2016, el cual incluye los términos MESH, MEDLINE y LILACS (http:// decs.bvs.br/E/homepagee.htm).

\section{RESUMEN}

INTRODUCCIÓN: El corazón es el órgano más comúnmente afectado por anormalidades congénitas, con una incidencia de 0.8 por cada 100 nacidos vivos. Cerca de dos tercios de todos los procedimientos son en la actualidad realizados antes del año de edad, lo que mejora la sobrevida y la calidad de vida. Este estudio busca determinar cuáles son las principales intervenciones quirúrgicas realizadas para tratar las cardiopatías congénitas y sus complicaciones.

MATERIALES Y MÉTODOS: Se realizó un estudio observacional, descriptivo de corte transversal; con 70 pacientes pediátricos diagnosticados y tratados quirúrgicamente por cardiopatías congénitas. Los datos fueron tomados de las historias clínicas mediante un formulario. El análisis estadístico se realizó utilizando el programa SPSS versión 15.

RESULTADOS: La mediana de la edad fue de 1.1 años, el 60\% fueron de sexo femenino. El 90\% de las cardiopatías fueron no cianógenas. El diagnóstico más frecuente fue la persistencia del conducto arterioso (58.57\%), seguido de la comunicación interventricular (12.86\%). Según el tipo de procedimiento el $58.57 \%$ se realizaron para cierre de persistencia del conducto arterioso y un $12.86 \%$ fueron reparaciones quirúrgicas para cierre de comunicación interventricular. La mediana de estadía en la unidad de cuidados intensivos fue de 4 días y la mediada de estadía en la sala general de 5 días. La principales complicaciones observadas en esta población pediátrica sometida a un procedimiento quirúrgico fueron: la neumonía (11.4\%) y la sepsis de origen no especificado (8.6\%).

CONCLUSIÓN: Los tratamientos para las cardiopatías congénitas se realizaron a edades tempranas (Media $=2.5 \pm 3.2$ años). Más de la mitad de los procedimientos quirúrgicos realizados para cardiopatías quirúrgicas fueron para corregir la persistencia del conducto arterioso y la principal complicación fue la neumonía.

PALABRAS CLAVE: CARDIOPATÍAS CONGÉNITAS, CIRUGÍA CARDÍACA, CATETERISMO CARDÍACO, PEDIATRÍA, COMPLICACIONES.

\section{ABSTRACT}

Congenital heart diseases, surgical treatment and complications in pediatric sample of Hospital Vicente Corral Moscoso, 2017-August 2019.

BACKGROUND: The heart is the most commonly affected organ by congenital diseases, with and incidence of 0.8 per 100 newborns. Nearly two thirds of all the surgical procedures are now a days performed before the first year of life, improving survival rate and life quality. This study aims to determine the frequency of the surgical interventions performed to treat congenital heart diseases and its complications.

METHODS: An observational, descriptive cross sectional study was carried out; with 70 pediatric patients diagnosed and surgically treated for congenital heart diseases. The data was collected from the patient's medical records using a form. Statistical analysis was performed using SPSS version 15 software.

RESULTS: The median age was 1.1 years, $60 \%$ of the sample were women. $90 \%$ of the heart diseases were non-cyanogenic. The most frequent diagnosis was: persistence of the arterial duct (58.57\%), followed by interventricular communication (12.86\%). The type of procedures corresponds to the heart disease, thus 58.57\% were performed for closure of arterial duct persistence and $12.86 \%$ were surgical repairs for closure of interventricular communication. The median stay in the intensive care unit was 4 days and the median stay in general hospitalization room was 5 days. The main complications in this pediatric population undergoing a surgical procedure were: pneumonia (11.4\%) and sepsis of unspecified origin (8.6\%).

CONCLUSION: Treatment for heart diseases were performed at early ages (average age $=2.5 \pm 3.2$ ). More than half of the surgical procedures for congenital heart disease were performed to correct the persistence of the ductus arteriosus, the main complication was pneumonia.

KEYWORDS: CONGENITAL HEART DEFECTS, CARDIAC SURGERY, CARDIAC CATHETERIZATION, PEDIATRICS, COMPLICATIONS. 


\section{INTRODUCCIÓN}

Las cardiomiopatías pediátricas afectan a una reducida porción de la población pediátrica, pero la constelación de desórdenes abarca un amplio rango de síntomas y grados de severidad de la enfermedad [1].

En el mundo nacen cada año 135 millones de niños y se estima que la prevalencia de las cardiopatías congénitas es de 0.5 a 9 por cada 1000 nacidos vivos, lo que resulta en 1.3 millones de recién nacidos en el mundo que tienen algún tipo de cardiopatía congénita [2]. En Latinoamérica nacen cada año 54000 niños con cardiopatías congénitas y de estos 41000 requieren tratamiento, pero solo 17000 son intervenidos. En Colombia, de 150 cirugías por millón de habitantes necesarias solo 52 intervenciones son realizadas por cada millón de habitantes [2]. En los Estados Unidos, cada año nacen aproximadamente 35000 niños con alguna enfermedad cardíaca congénita; de los cuales aproximadamente 10000 niños tienen una condición severa que requiere de una cirugía cardíaca antes del primer año de vida [3].

El corazón es el órgano más comúnmente afectado por anormalidades congénitas, con una incidencia de 0.8 por cada 100 nacidos vivos. Aunque comunes, la mayoría de las condiciones son menores y no requieren intervención quirúrgica [4] . Cada vez es mayor la tendencia a reparar las lesiones a edades más tempranas y conseguir una reparación anatómica completa en la infancia, en lugar de realizar una serie de procedimientos en etapas. Cerca de dos tercios de todos los procedimientos son en la actualidad realizados antes del año de edad [4].

Los objetivos de la rehabilitación cardíaca pediátrica están dirigidos a mejorar la capacidad funcional del niño, la calidad de vida, incrementar la masa magra en comparación a la masa grasa, incrementar la capacidad para realizar actividad física, educar a la familia y al niño para adoptar estilos de vida saludables, manejo y reconocimiento de los síntomas, y disminuir el riesgo de enfermedad cardiovascular futura. [1]

Los tratamientos actuales para las cardiomiopatías están enfocados en mejorar la función cardíaca, prevenir o controlar las arritmias y minimizar los síntomas cardíacos; pero el trasplante cardíaco es el tratamiento definitivo para las enfermedades progresivas [1]. La técnica quirúrgica tiene fuerte asociación con los resultados del paciente [3]. Los dispositivos de apoyo circulatorio mecánico, en los pacientes pediátricos, tienen un rol importante en la práctica futura de la cirugía cardíaca congénita [5].

Algunos ejemplos de lesiones comúnmente reparadas son: la tetralogía de Fallot, que es la cardiopatía cianótica más común y representa el 5-10\% de todas las enfermedades cardíacas congénitas; el procedimiento quirúrgico restaura la anatomía normal y fisiológica y está asociado con un 98-99\% de sobrevida en la época actual [4]. La cirugía de reparación de la transposición de los grandes vasos debe planificarse en las primeras semanas de vida, si la cirugía se retrasa por mucho tiempo entonces el ventrículo izquierdo se torna demasiado débil para soportar la circulación sistémica [4]. Otro defecto es la circulación funcionalmente univentricular izquierda que se caracteriza por que uno de los ventrículos está subdesarrollado y es incapaz de soportar la circulación total; esta condición rara representa el 3-5\% de todas las enfermedades cardíacas congénitas; la mayoría de estos casos requieren de una serie de intervenciones planificadas que adaptan el corazón y la circulación a trabajar con una circulación ventricular única [4].

La cateterización cardíaca intervencionista se ha desarrollado dramáticamente durante los últimos 20 años de tal manera que ahora es posible cerrar la mayoría de los defectos septales y la persistencia del conducto arterioso a través de la vena femoral. Las nuevas técnicas quirúrgicas ofrecen la posibilidad de reemplazos de válvulas percutáneas en niños mayores y hay un creciente interés en los procedimientos híbridos, en los cuales tanto el cirujano como el cardiólogo intervencionista trabajan juntos, utilizando técnicas quirúrgicas para proveer acceso para el acceso de los stents y dispositivos, a menudo directamente al corazón [4] [6].

Los resultados en la cirugía cardíaca pediátrica han mejorado dramáticamente desde las primeras cirugías hace 40 años. La mortalidad ha disminuido desde el $90-100 \%$ en sus inicios a un $4 \%$ para los casos de alta complejidad, y virtualmente no existe riesgo de mortalidad para los defectos cardíacos simples. Parte de estas mejoras se deben a los avances en la anestesia cardíaca pediátrica, el bypass cardiopulmonar pediátrico y el desarrollo de unidades de cuidados intensivos pediátricos, por lo cual los resultados dependen en gran medida de la calidad de la técnica de la reparación quirúrgica, entre otros aspectos [3] [7] [8].

Aunque los procedimientos quirúrgicos cardíacos son muy recientes en el Hospital Vicente Corral Moscoso, son cirugías que, en la actualidad, se realizan con bastante frecuencia. De allí, que este estudio busca determinar cuáles son las principales intervenciones que se realizan para tratar este tipo de patologías, las principales técnicas empleadas y las complicaciones.

\section{MATERIALES Y MÉTODOS}

Se realizó un estudio observacional, descriptivo de corte transversal, cuyo universo estuvo conformado por los pacientes con cardiopatías congénitas, sometidos a cirugía cardiaca en el Hospital Vicente Corral Moscoso durante el periodo 2017agosto 2019. Se estudió a la totalidad del universo. Los criterios de inclusión fueron; población pediátrica hasta los ocho años, con diagnóstico de cardiopatía congénita, que fueron intervenidos quirúrgicamente; como criterio de exclusión se consideró historias clínicas con datos incompletos. Se incluyeron en el estudio un total de 70 niños.

Se revisó las historias clínicas de los pacientes de la población de estudio y para la recolección de los datos se elaboró un formulario con las variables de interés según el propósito del estudio: edad, sexo, tipo de cardiopatía congénita, tratamiento quirúrgico o procedimiento de cateterismo realizado y finalmente si se presentó alguna complicación. Los datos fueron transcritos de los formularios a una base digital y analizados con el programa SPSS versión 15. Los resultados se presentan con frecuencias y porcentajes y medidas de tendencia central por medio de tablas.

\section{RESULTADOS}

Se observó un porcentaje de $27.1 \%$ de pacientes con edades entre los 2-11 meses y el mismo porcentaje de pacientes de 1-2 
años. El promedio de edad fue de $2.5 \pm 3.2$ años, la mediana de 1.1 años. Según el sexo, el $60 \%$ de la población es del sexo femenino. Los pacientes proceden principalmente de la provincia del Azuay (78.6\%) y del Cañar (10.0\%) (Tabla 1).

Tabla 1. Distribución de 70 pacientes pediátricos sometidos a tratamiento quirúrgico por cardiopatía congénita en el Hospital Vicente Corral Moscoso durante el año 2017 - agosto 2019, según la edad, sexo y residencia.

\begin{tabular}{|c|c|c|}
\hline Variable & Frecuencia & Porcentaje \\
\hline \multicolumn{3}{|l|}{ Edad } \\
\hline 0-1 mes & 12 & 17.1 \\
\hline 2-11 meses & 19 & 27.1 \\
\hline $12-24$ meses & 19 & 27.1 \\
\hline 25-60 meses & 12 & 17.1 \\
\hline$>60$ meses & 8 & 11.4 \\
\hline \multicolumn{3}{|l|}{ Sexo } \\
\hline Femenino & 42 & 60.0 \\
\hline Masculino & 28 & 40.0 \\
\hline \multicolumn{3}{|l|}{ Procedencia } \\
\hline Azuay & 55 & 78.6 \\
\hline Cañar & 7 & 10.0 \\
\hline Loja & 3 & 4.3 \\
\hline Santo Domingo & 1 & 1.4 \\
\hline $\begin{array}{r}\text { Zamora Chin- } \\
\text { chipe }\end{array}$ & 1 & 1.4 \\
\hline El Oro & 1 & 1.4 \\
\hline Morona Santiago & 2 & 2.9 \\
\hline
\end{tabular}

Fuente: Formulario de datos.

Elaboración: Los autores.

Tabla 2. Distribución de 70 pacientes pediátricos sometidos a tratamiento quirúrgico por cardiopatía congénita en el Hospital Vicente Corral Moscoso durante el año 2017 - agosto 2019, según el tipo de cardiopatía.

\begin{tabular}{|c|c|c|}
\hline $\begin{array}{c}\text { Tipo de } \\
\text { Cardiopatía }\end{array}$ & Frecuencia & Porcentaje \\
\hline Cianógena & 7 & 10.0 \\
\hline No cianógena & 63 & 90.0 \\
\hline
\end{tabular}

Fuente: Formulario de datos. Elaboración: Los autores.

La mayoría de las cardiopatías (90\%) fueron de tipo no cianógena, en comparación al $10 \%$ de cardiopatías de tipo cianógena (Tabla 2).

Más de la mitad de las cardiopatías (58.57\%) que se trataron en el Hospital Vicente Corral Moscoso fueron por persistencia del conducto arterioso, en menor frecuencia fueron por comunicación interventricular (12.86\%) y comunicación interauricular (7.14\%) (Tabla 3).Los principales procedimientos quirúrgicos realizados fueron cierre de persistencia del conducto arterioso (58.57\%) y reparación quirúrgica de comunicación interventricular (12.86\%). El $70.0 \%$ de procedimientos se realizaron sin circulación extracorpórea (Tabla 4).
Tabla 3. Distribución de 70 pacientes pediátricos sometidos a tratamiento quirúrgico por cardiopatía congénita en el Hospital Vicente Corral Moscoso durante el año 2017 - agosto 2019, según el diagnóstico.

\begin{tabular}{|l|c|c|}
\hline Diagnóstico & $\mathbf{n}$ & $\%$ \\
\hline Persistencia conducto arterioso & 41 & 58.57 \\
\hline Comunicación interventricular & 9 & 12.86 \\
\hline Comunicación interauricular & 5 & 7.14 \\
\hline Atresia pulmonar & 2 & 2.86 \\
\hline CIA+CIV & 2 & 2.86 \\
\hline Comunicación auriculo ventricular & 2 & 2.86 \\
\hline DATVP & 2 & 2.86 \\
\hline Tetralogía de Fallot & 2 & 2.86 \\
\hline Estenosis pulmonar severa & 1 & 1.43 \\
\hline Insuficiencia mitral severa & 1 & 1.43 \\
\hline ALCAPA & 1 & 1.43 \\
\hline Arco aórtico interrumpido & 1 & 1.43 \\
\hline Atresia tricúspidea + CIV + Estenosis & 1 & 1.43 \\
\hline pulmonar & & \\
\hline A & &
\end{tabular}

ALCAPA: Arteria Coronaria Izquierda Anómala desde la Arteria Pulmonar, CIV: Comunicación Interventricular, CIA: Comunicación Interauricular, DATVP: Drenaje anómalo total de venas pulmonares.

Fuente: Formulario de datos.

Elaboración: Los autores.

Tabla 4. Distribución de 70 pacientes pediátricos sometidos a tratamiento quirúrgico por cardiopatía congénita en el Hospital Vicente Corral Moscoso durante el año 2017 - agosto 2019, según el tipo de procedimiento quirúrgico.

\begin{tabular}{|l|c|c|}
\hline Tipo de procedimiento & n & $\%$ \\
\hline $\begin{array}{l}\text { Cierre de persistencia del conducto arterioso } \\
\text { Reparación quirúrgica de comunicación }\end{array}$ & 41 & 58.57 \\
\hline $\begin{array}{l}\text { interventricular } \\
\text { Reparación quirúrgica de comunicación } \\
\text { interauricular e interventricular con parche }\end{array}$ & 5 & 12.86 \\
\hline $\begin{array}{l}\text { Fístula de Blalock-tauswsing izquierda } \\
\text { Corrección de drenaje anómalo total de venas } \\
\text { pulmonares }\end{array}$ & 2 & 2.14 \\
\hline $\begin{array}{l}\text { Reparación de comunicación } \\
\text { auriculoventricular }\end{array}$ & 2.86 \\
\hline $\begin{array}{l}\text { Reparación quirúrgica de tetralogía de Fallot } \\
\text { Plastia de la válvula pulmonar }\end{array}$ & 2 & 2.86 \\
\hline $\begin{array}{l}\text { Cerclaje de arteria pulmonar } \\
\text { Cierre de ductus, anastomosis subclavia } \\
\text { carotidea con aorta término terminal }\end{array}$ & 1 & 1.43 \\
\hline $\begin{array}{l}\text { Reparación de arteria coronaria izquierda } \\
\text { anómala desde arteria pulmonar ALCAPA con } \\
\text { técnica de Takeuchi }\end{array}$ & 1 & 1.43 \\
\hline $\begin{array}{l}\text { Reparación quirúrgica de comunicación i } \\
\text { nterauricular con parche }\end{array}$ & 1 & 1.43 \\
\hline $\begin{array}{l}\text { Reparación de hendidura mitral más } \\
\text { anuloplastia }\end{array}$ & 1 & 1.43 \\
\hline & & \\
\hline & 1 & \\
\hline & & \\
\hline
\end{tabular}

Fuente: Formulario de datos.

Elaboración: Los autores. 
Tabla 5. Distribución de 70 pacientes pediátricos sometidos a tratamiento quirúrgico por cardiopatía congénita en el Hospital

Vicente Corral Moscoso durante el año 2017 - agosto 2019, según las complicaciones.

\begin{tabular}{|l|c|c|}
\hline Complicaciones & $\mathbf{n}$ & $\%$ \\
\hline Ninguna & 40 & 57.1 \\
\hline Neumonía & 8 & 11.4 \\
\hline Otras & 7 & 10.0 \\
\hline Sepsis & 6 & 8.6 \\
\hline Fallece & 4 & 5.7 \\
\hline Enfisema & 2 & 2.9 \\
\hline Atelectasia & 1 & 1.4 \\
\hline Choque & 1 & 1.4 \\
\hline cardiogénico & 1 & 1.4 \\
\hline ITU + Sd. Febril & & \\
\hline
\end{tabular}

*Otras (Quilotórax, síndrome de distres respiratorio, quemadura por manta)

Fuente: Formulario de datos.

Elaboración: Los autores.

Las complicaciones más frecuentes fueron la neumonía (11.4\%) y la sepsis (8.6\%). El 5.7\%(n=4) de los pacientes fallecieron durante o después de la cirugía (Tabla 5).

Tabla 6. Distribución de 70 pacientes pediátricos sometidos a tratamientos quirúrgicos por cardiopatías congénitas en el Hospital Vicente Corral Moscoso, durante el año 2017 - agosto 2019, según días de permanencia.

\begin{tabular}{|l|c|c|}
\hline Variables & $\mathbf{n}$ & $\%$ \\
\hline Días en la UCl* & & \\
\hline 1-2 días & 20 & 28.6 \\
\hline 3-7 días & 27 & 38.6 \\
\hline 8-14 días & 8 & 11.4 \\
\hline 15-30 días & 5 & 7.1 \\
\hline 31-60 días & 6 & 8.6 \\
\hline >60 días & 4 & 5.7 \\
\hline Días en la sala general** & & \\
\hline 3-7 días & 33 & 47.1 \\
\hline 0-2 días & 16 & 22.9 \\
\hline 8-14 días & 11 & 15.7 \\
\hline >14 días & 10 & 14.3 \\
\hline
\end{tabular}

*Días UCl: promedio $10.9 \pm 19.1$ mediana 4 días.

**Días en la sala general: $6.7 \pm 8.7$ mediana 5 días

Fuente: Formulario de datos.

Elaboración: Los autores.

El mayor porcentaje de niños (38.6\%) permanecieron entre 3-7 días en la unidad de cuidados intensivos; el promedio de estancia fue de $10.9 \pm 19.1$ y la mediana de 4 días. La permanencia en la sala general, para la mayoría de la población (47.1\%) fue de 3-7 días, con un promedio de: $6.7 \pm 8.7$ mediana 5 días (Tabla 6).

\section{DISCUSIÓN}

La prevalencia estimada de cardiopatías congénitas al nacimiento es de 6 y 10 casos por cada 1000 nacidos vivos, pero con el diagnóstico y tratamiento precoz el $90 \%$ de los casos sobreviven hasta la vida adulta[9]. Para Martínez Quintana las cardiopatías congénitas continúan incrementándose por la edad gestacional tardía, el uso de nuevas técnicas de fecundación, cambios fisiológicos extremos del embarazo, consumo de sustancias y drogas, medicamentos, entre otras [10].

Se identificó, en el presente estudio, que el grupo etario más frecuente de los pacientes con cardiopatías congénitas fue de 2-11 meses y de $1-2$ años con un $27.1 \%$ para cada grupo. Las cardiopatías predominaron en el sexo femenino (60.0\%). Las principales cardiopatías fueron la persistencia del conducto arterioso (58.57\%) y la comunicación interventricular (12.86\%).

A diferencia de nuestros resultados, García y cols., en Colombia, en el año 2016, al analizar 5900 casos con estudios de ecocardiograma, evidenciaron que el $56.1 \%$ presentaron cardiopatías congénitas, $54.3 \%$ se presentaron en el sexo masculino. Las cardiopatías más frecuentes y en orden de frecuencia fueron comunicación interventricular, obstrucción del tracto de salida del ventrículo derecho, comunicación interauricular, ductus arterioso persistente y obstrucción de salida del ventrículo izquierdo [11]. Es importante mencionar que para el presente estudio se consideraron únicamente las cardiopatías que fueron tratadas quirúrgicamente.

Según el estudio de Duque y cols., en Colombia, durante el 2018; existe asociación entre el número de hijos con cardiopatías y factores sociodemográficos como el estrato socioeconómico, el nivel de educación de los padres, la zona de residencia, el tipo de vivienda; y en cuanto a los factores de riesgo preconcepcionales, se encontró asociación estadísticamente significativa entre el número de hijos con cardiopatías y la exposición a fertilizantes, a combustibles, consumo de medicamentos antihipertensivos, consumo de alcohol y cocaína [12].

La frecuencia de comunicación interauricular tratada en este estudio fue del $7.14 \%$, la comunicación interventricular del $12.86 \%$, persistencia del conducto arterioso $58.57 \%$, estenosis pulmonar $1.43 \%$ y tetralogía de Fallot $2.86 \%$; datos que difieren de los reportados por Pérez Lescure y cols., en el 2017 (España), quienes observaron una incidencia de comunicación interauricular del $6.31 \%$, comunicación interventricular del 3.48\%, ductus arterioso persistente del $2.47 \%$, coartación de aorta del $0.55 \%$, estenosis pulmonar del $0.5 \%$, trasposición de grandes vasos de $0.49 \%$, canal auriculoventricular del $0.45 \%$ y tetralogía de Fallot de $0.41 \%$. [13]

El porcentaje de pacientes que fallecieron por la condición de la cardiopatía y el procedimiento quirúrgico en nuestro estudio fue del $5.7 \%$, cifras que superan al $4 \%$ reportado por Becker Rencoret, en Chile, en el 2016, en pacientes intervenidos por síndrome de hipoplasia de Corazón Izquierdo. La estancia hospitalaria es de 17 días y la sobrevida es de 8 años aproximadamente [14]. En nuestro estudio la mediana de estadía en la unidad de cuidados intensivos fue de 4 días y en la sala general fue de 5 días.

En cuanto a las técnicas o procedimientos quirúrgicos empleados, estos se corresponden en frecuencia con el tipo de cardiopatía. Así, 
por ejemplo, el procedimiento de cierre de persistencia del conducto arterioso representó el $58.57 \%$ de las intervenciones realizadas en la población pediátrica del Hospital Vicente Corral Moscoso. La mortalidad registrada en nuestro estudio es de apenas el $5.7 \%$.

Entre los pacientes con atresia pulmonar con comunicación interventricular, los pacientes con un sistema arterial pulmonar adecuado y sin arteria colateral aortopulmonar mayor( MAPCA) tienen resultados similares a los de la tetralogía de Fallot, pero un 35\% de casos que requieren procedimientos complejos pueden presentar una mortalidad precoz. En cuanto a la supervivencia a largo plazo, varios parámetros influyen en ella; la mortalidad tardía dependerá de la presencia de factores tales como: reintervenciones, arritmias, estenosis pulmonares, fallo cardiaco, complicaciones neurológicas y endocarditis [15]. Centella Hernández et al., menciona también que la edad es un factor que influye directamente en la incidencia de la recuperación de estos pacientes, de aquellos que fueron intervenidos antes de los 5 años, solo el $10 \%$ de casos necesitaran una reintervención a los 10 años. La historia natural de la atresia pulmonar varía debido a la gran variedad anatómica de esta cardiopatía; los casos que presentan ramas pulmonares levemente hipoplásicas, pueden vivir $\sin$ tratamiento los primeros 6 meses de vida y solo el $10 \%$ sobrevive más de un año; los pacientes con esta cardiopatía que tienen alguna colateral aortopulmonar, la mitad fallecen antes de los 3 años de vida y el $90 \%$ antes de los 10 años y un tercer grupo de pacientes, los que presentan gran número de colaterales, subcianosis y aumento de flujo pulmonar, pueden vivir hasta la edad adulta y desencadenar en ocasiones una hipertensión pulmonar severa que da paso al síndrome de Eisenmenger[15] .

La comunicación interauricular (CIA) más frecuente es el ostium secundum y puede ser cerrada por vía percutánea o en el quirófano, después de su cierre se debe estar seguro de que las venas pulmonares drenen en la aurícula izquierda y las cavas en la derecha. La de menor frecuencia es la CIA tipo seno venoso, su tratamiento consiste en la colocación de un parche que rodea la CIA y el orificio de drenaje venoso anómalo[16]. La CIV es una cardiopatía frecuente tanto aislada como asociada a otras malformaciones, su tratamiento consiste en la colocación de un parche, siendo más común la CIV peri membranosa y menos común la CIV subarterial [16].

Los estudios de Galian Gay y cols., en el 2019 reportan que en los casos de estenosis de la válvula pulmonar, tras el tratamiento con valvuloplastia percutánea en un 38\% de los casos se requiriere de una reintervención. Al menos $12 \%$ de los pacientes presentan una de las siguientes complicaciones: en un $8.2 \%$ arritmias supraventriculares, $3.8 \%$ insuficiencia cardiaca, $3.2 \%$ accidente cerebrovascular y en el $0.6 \%$ fallecimiento, tromboembolia y arritmia ventricular respectivamente [17]. A diferencia de estos datos la principal complicación en nuestro estudio fue la neumonía con un $11.4 \%$, seguido de la sepsis con un $8.6 \%$.

Ventajosamente, la mayoría de los pacientes sometidos a algún procedimiento quirúrgico por una cardiopatía en el hospital Vicente Corral Moscoso son a edades tempranas, con una mediana de 1.1 años, lo cual implica que la sobrevida será mayor, y requerirán probablemente menos tratamientos invasivos a futuro para mejorar su calidad de vida.

\section{CONCLUSIÓN}

Los tratamientos para las cardiopatías congénitas se realizaron a edades tempranas (Media $=2.5 \pm 3.2$ años). La mayoría de las cardiopatías (90\%) fueron de tipo no cianógeno. Las cardiopatías más frecuentes fueron la persistencia del conducto arterioso y la comunicación interventricular. Los procedimientos más realizados fueron el cierre de la persistencia del conducto arterioso y la reparación quirúrgica de cierre de la comunicación interventricular. La mediana de estadía en la unidad de cuidados intensivos fue de 4 días y la mediana de estadía en la sala general fue de 5 días. Entre las complicaciones más frecuentes estuvieron la neumonía y la sepsis.

\section{ABREVIATURAS}

ALCAPA: Arteria Coronaria Izquierda Anómala desde la Arteria Pulmonar; AV: Aurículo ventricular; CIV: Comunicación Interventricular; CIA: Comunicación Interauricular; DATVP: Drenaje anómalo total de venas pulmonares.

\section{AGRADECIMIENTOS}

A todos quienes contribuyeron y facilitaron la ejecución de esta investigación, de manera especial a la Universidad Católica de Cuenca.

\section{FINANCIAMIENTO}

El estudio se financió con recursos propios de los autores.

\section{DISPONIBILIDAD DE DATOS Y MATERIALES}

Los datos fueron obtenidos de las historias clínicas de los pacientes con la autorización del Departamento de Docencia y de las Autoridades del Hospital Vicente Corral Moscoso; la revisión bibliográfica se realizó en base a artículos científicos de bases de datos digitales.

\section{CONTRIBUCIÓN DE LOS AUTORES}

JP: redacción del manuscrito y análisis de los datos, LM, EP, SP: recolección de datos y elaboración del manuscrito, CA: concepción y análisis crítico del artículo.

\section{INFORMACIÓN DE LOS AUTORES}

- Jennifer Pamela Picón Rodríguez. Médico General, Clínica Latinoamericana. (D) ORCID: https://orcid.org/ 0000-0003-4264-6208

- Luis Enrique Marcano Sanz. Especialista de Segundo Grado en Cirugía Pediátrica y Cardiovascular, Máster en Urgencias Médicas, Profesor Asistente de posgrados de la Universidad de Cuenca. Servicio de Cardiología Pediatrica, Hospital Vicente Corral Moscoso. (D) ORCID: https:// orcid.org/ 0000-0003-3811-1120

- Erika Aracely Pinos Vélez. Médico General, Clínica Latinoamericana. (D) ORCID: https://orcid.org/ 0000-0002-0932-9991

- Susana Janeth Peña Cordero. Doctora en Medicina y Cirugía, Especialista en Medicina Interna, Decana de la Unidad de Salud y Bienestar de la Universidad Católica de Cuenca. (D) ORCID: https://orcid.org/ 0000-0002-6526-2437

- Carlos Eduardo Arévalo Peláez. Doctor en Medicina y Cirugía. Docente de la Universidad Católica de Cuenca y Universidad de Cuenca. (D) ORCID: https://orcid.org/0000-0002-6427-4954 


\section{CONFLICTOS DE INTERÉS}

Los autores no reportan ningún conflicto de interés. El presente artículo se basa en el trabajo de titulación de la carrera de medicina, de la Universidad Católica de Cuenca, disponible en el repositorio digital de la misma.

\section{CONSENTIMIENTO PARA PUBLICAR}

Todos los autores autorizan la publicación de este artículo.

\section{APROBACIÓN DE ÉTICA Y CONSENTIMIENTO DE PARTICIPACIÓN}

Los autores cuentan con la autorización ética y las aprobaciones correspondientes de la Institución donde se realizó el estudio.

\section{CÓMO CITAR ESTE ARTÍCULO:}

Picón J, Marcano L, Pinos E, Peña S, Arévalo C. Cardiopatías congénitas, tratamiento quirúrgico y sus complicaciones en población pediátrica del Hospital Vicente Corral Moscoso, 2017- Agosto 2019. Rev Med HJCA 2021; 13(1): 21-26. DOI: http://dx.doi.org/10.14410/2021.13.1.ao.03

\section{PUBLONS}

P Contribuye con tu revisión en: https://publons.com/publon/49419435/

\section{REFERENCIAS BIBLIOGRÁFICAS}

1. Thrush PT, Vogel C. Cardiac rehabilitation in pediatric cardiomyopathy. Prog Pediatr Cardiol. 2018;49:43-46. DOI: https://doi.org/10.1016/j.ppedcard.2018.04.001

2. Sandoval N. Cardiopatías congénitas en Colombia y en el mundo. Rev Colomb Cardiol. 2015;22(1):1-2. DOI: https://doi.org/10.1016/j.rccar.2015.03.004

3. Karamichalis JM, Barach PR, Nathan M, Henaine R, del Nido PJ, Bacha EA Assessment of technical competency in pediatric cardiac surgery. Prog Pediatr Cardiol. 2012;33(1):15-20. DOI: https://doi.org/10.1016/j.ppedcard.2011.12.003

4. Barron DJ, Jones TJ, Mussa S. Essentials of paediatric cardiac surgery. Surg Oxf. 2018;36(2):91-96. DOI: https://doi.org/10.1016/j.mpsur.2017.11.005

5. Cohen G, Permut L. Decision making for mechanical cardiac assist in pediatric cardiac surgery. Semin Thorac Cardiovasc Surg Pediatr Card Surg Annu. 2005;8(1):41-50. DOI: https://doi.org/10.1053/j.pcsu.2005.02.004

6. Staudt GE, Eagle SS, Hughes AK, Donahue BS. Evaluation of Dynamic Ultrasound for Arterial Access in Children Undergoing Cardiac Surgery. J Cardiothorac Vasc Anesth. 2019;33(7):1926-1929. DOI: https://doi.org/10.1053/j. jvca.2018.11.050

7. Alam S, Shalini A, Hegde RG, Mazahir R, Jain A. A comparative study of the risk stratification models for pediatric cardiac surgery. Egypt J Crit Care Med. 2018;6(1):5-8. DOI: https://doi.org/10.1016/j.ejccm.2018.03.001

8. Konfirst C, Preston S, Yeh T. Checklists and Safety in Pediatric Cardiac Surgery. Semin Thorac Cardiovasc Surg Pediatr Card Surg Annu. 2015;18(1):43-50. DOI: https://doi.org/10.1053/j.pcsu.2015.01.006

9. Sánchez Fernández PL, Martín García A, García Cuenllas L, Plata B, Maroto E. Cardiopatías congénitas. Med - Programa Form Médica Contin Acreditado. 2017;12(45):2683-2699. DOI: https://doi.org/10.1016/j.med.2017.11.007

10. Martínez-Quintana E, Romero-Requejo A, Rodríguez-González F. Cardiopatías congénitas y embarazo. Clínica E Investig En Ginecol Obstet. 2016;43(1):24-31. DOI: https://doi.org/10.1016/j.gine.2014.11.002
11. García A Caicedo M, Moreno K, Sandoval N, Ronderos M, Dennis R. Diferencias regionales en cardiopatías congénitas. Rev Colomb Cardiol. 2017;24(2):161168.DOI: https://doi.org/10.1016/j.rccar.2016.06.012

12. Duque PA, Valencia Rico CL, Araujo JJ. Factores sociodemográficos y factores de riesgo preconcepcionales en padres y madres de niños con cardiopatías congénitas. Enferm Clínica. 2018;28(5):300-308. DOI: https://doi.org/10.1016/j. enfcli.2018.03.003

13. Pérez-Lescure Picarzo J, Mosquera González M, Latasa Zamalloa P, Crespo Marcos D. Incidencia y evolución de las cardiopatías congénitas en España durante 10 años (2003-2012). An Pediatría. 2018;89(5):294-301. DOI: https://doi. org/10.1016/j.anpedi.2017.12.009

14. Becker Rencoret P, Besa Bandeira S, Riveros González S, Frangini Sanhueza P, Springmüller Pinto D, González Foretic R, et al. Resultados comparativos de cirugía de Fontan en pacientes con y sin hipoplasia de corazón izquierdo. Rev Chil Pediatría. 2016;87(5):366-72. DOI: https://doi.org/10.1016/j.rchipe.2016.03.001

15. Centella Hernández T, Stanescu D, Stanescu S. Atresia pulmonar con comunicación interventricular. Cir Cardiovasc. 2014;21(2):132-137. DOI: https://doi. org/10.1016/j.circv.2014.02.003

16. Gil-Jaurena JM, González-López M. Comunicación interauricular. Comunicación interventricular. Canal aurículo-ventriculary Ventana aorto-pulmonar. Cir Cardiovasc. 2014;21(2):86-89.DOI: https://doi.org/10.1016/j.circv.2014.03.006

17. Galian-Gay L, Gordon B, Marsal JR, Rafecas A, Pijuan Domènech A, Castro MA, et al. Determinants of long-term outcome of repaired pulmonary valve stenosis. Rev Esp Cardiol Engl Ed. 2020; 73(2):131-138. DOI:https://doi.org/10.1016/j. rec.2019.02.014 Martínez-Quintana E, Romero-Requejo A, Rodríguez-González F. Cardiopatías congénitas y embarazo. Clínica E Investig En Ginecol Obstet. 